\title{
COMPENSATION MODELS IN CHLORINE DOPED CdTe BASED ON POSITRON ANNIHILATION AND PHOTOLUMINESCENCE SPECTROSCOPY*
}

\author{
W. Stadler, D.M. Hofmann, B.K. Meyer
}

Technische Universität München, Physik Department, E16, 85747 Garching, Germany

R. Krause-Rehberg, A. Polity, Th. Abgarjan

Martin-Luther-Universität Halle-Wittenberg, FB Physik, 06108 Halle, Germany

M. SalK, K.W. BENZ

Kristallographisches Institut, Universität Freiburg, 79104 Freiburg, Germany

and M. Azoulay

Soreq, Nuclear Research Centre, Yavne 70600, Israel

In this investigation positron annihilation, photoluminescence and electron paramagnetic resonance techniques are employed to gain insight in the compensation of CdTe doped with the halogen $\mathrm{Cl}$. We will demonstrate that the high resistivity of $\mathrm{CdTe}: \mathrm{Cl}$ cannot be explained by the interaction between the shallow effective mass type donor $\mathrm{Cl}$ on Te site and the doping induced shallow acceptor complex, a Cd vacancy paired off with a nearest-neighbour $\mathrm{Cl}$ atom ( $A$ centre). From electron paramagnetic resonance investigations we conclude that the mid gap trap, often detected by electrical methods in CdTe, is not the isolated Cd vacancy.

PACS numbers: 78.70.Bj, 71.55.Gs, 78.55.Et

The atomistic structure identification of point defects present in stoichiometric or non-stoichiometric $\mathrm{CdTe}$ is mainly based on electron paramagnetic resonance (EPR) techniques. EPR makes it possible to determine the microscopic structure and the concentration of the defect under investigation. The positions of the energy levels of the defect can be measured by illuminating the sample with monochromatic light during the EPR experiment (photo-EPR). As a quantitative method, EPR can be used for comparison with Hall or positron annihilation data and confirm whether or not a certain compensation model is correct. Based on EPR and photo-EPR, both the isolated Cd and Te vacancies were identified in CdTe [1, 2].

*This work was supported by the German Research Foundation DFG under contract Me 898/5-2. 
The determination of the Cd-vacancy and the Te-vacancy concentration based on EPR gave $6 \times 10^{15} \mathrm{~cm}^{-3}$ and $1 \times 10^{16} \mathrm{~cm}^{-3}$ as upper limits, respectively. The $V_{\mathrm{Cd}}^{2-l-}$ level position lies at $E<E_{\mathrm{VB}}+0.47 \mathrm{eV}$, together with its low concentration, this cannot explain the high resistivity of $n$ doped CdTe.

An energy level at $0.78 \mathrm{eV}$ in CdTe determined by electrical measurements (e.g. photoinduced current transient spectroscopy) was often attributed to the $V_{\mathrm{Cd}}^{2-1-}$ level [3], this is in contradiction to the EPR results. Pinning the Fermi level at mid gap to produce high resistive $\mathrm{CdTe}$ doped with $\mathrm{Cl}$ must have a different origin.

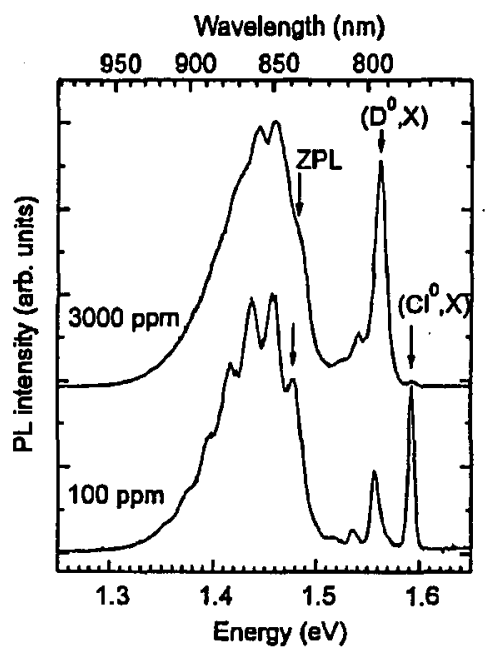

Fig. 1. Low-temperature PL spectra of CdTe samples doped with $100 \mathrm{ppm}$ and $3000 \mathrm{ppm}$ chlorine. The zero phonon line of the DAP recombination $(1.47 \mathrm{eV}$ band) involving the $A$ centres is marked with ZPL. At high doping level the phonon structure of the $1.47 \mathrm{eV}$ band is partially washed out due to the higher defect concentrations and the ZPL is slightly shifted to higher energies.

We investigated a series of samples doped to different levels with $\mathrm{Cl}$ by photoluminescence (PL) and positron annihilation (PA). In PL (see Fig. 1) at low doping level the exciton bound to the neutral $\mathrm{Cl}$ donor appears in equal intensity compared to the donor-acceptor-pair (DAP) transition at $1.47 \mathrm{eV}$. This DAP band is due to the recombination between isolated $\mathrm{Cl}$ on Te sites (shallow effective-mass donors) and the chlorine $A$ centre (acceptors), a $\mathrm{Cd}$ vacancy paired off with a nearest-neighbour $\mathrm{Cl}$. The microscopic structure of the $A$ centres was established by optically detected magnetic resonance (ODMR) experiments [4].

A method which is sensitive to neutral or negatively charged vacancies is the positron annihilation spectroscopy. If there are no trapping centres in the sample, a one-component positron lifetime spectrum is obtained with the bulk lifetime $\tau_{b}$, for CdTe $282 \mathrm{ps.} \mathrm{Open} \mathrm{volume} \mathrm{defects} \mathrm{lead} \mathrm{to} \mathrm{an} \mathrm{increase} \mathrm{in} \mathrm{the} \mathrm{trapping} \mathrm{time}$ due to the locally attractive potential of the defect, since the electron density in 
such a defect is lower compared to the bulk. The spectrum thus consists of two exponential lifetimes, the longer one $\tau_{2}=330 \mathrm{ps}$ (defect lifetime) typical for $\mathrm{Cd}$ mono-vacancies in CdTe [5].

The vacancy concentration $C_{\mathrm{v}}$ can be calculated

$$
C_{\mathrm{v}}=\frac{\kappa_{\mathrm{v}}}{\mu} \text { with } \kappa_{\mathrm{v}}=\frac{1}{\tau_{\mathrm{b}}} \frac{\bar{\tau}-\tau_{\mathrm{b}}}{\tau_{2}-\bar{\tau}} .
$$

$\mu$ is the trapping coefficient and $\bar{\tau}$ is the average lifetime calculated from the lifetime components by $\bar{\tau}=\sum I_{i} \tau_{i}$ with the relative intensities $I_{i}$. The series of CdTe: $\mathrm{Cl}$ samples was measured as a function of the temperature in the range from 14 to $350 \mathrm{~K}$ (see Fig. 2). The undoped sample did not show any positron trapping due to a vacancy-type defect. The positron lifetime spectrum showed the bulk lifetime. With increasing $\mathrm{Cl}$ contents the average lifetime increases and reaches a value of $352 \mathrm{ps}$ at room temperature for the highest doping concentration $(3000 \mathrm{ppm})$. For the $100 \mathrm{ppm}$ doped CdTe $\tau_{2}$ was $348 \mathrm{ps}$ slightly higher than found for the isolated mono-vacancy. Since the long lifetime only shows up in connection with the $\mathrm{Cl}$ doping we attribute it to the presence of $A$ centres as already evidenced by the PL measurements. For the determination of the respective concentration the trapping coefficient has to be known. We used $\mu=9 \times 10^{14} \mathrm{~s}^{-1}$ a value established for $\mathrm{Hg}$ vacancies in $\mathrm{HgCdTe}$. For further details see Ref. [6]. The resulting defect concentrations as a function of $\mathrm{Cl}$ doping are summarized in Table together with the values for the $\mathrm{Cl}$ concentration added to the melt and the $\mathrm{Cl}$ concentration incorporated into the crystal.

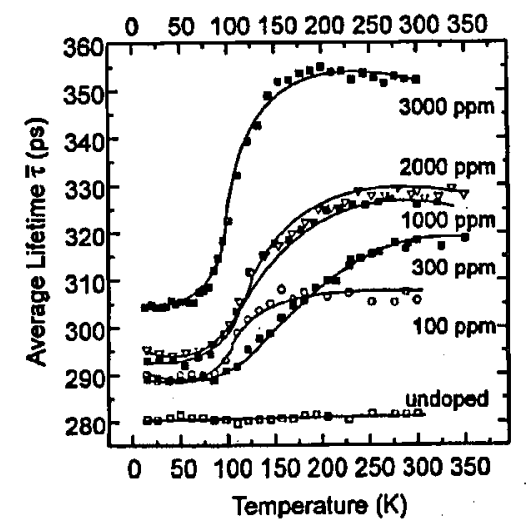

Fig. 2. Lifetime of the CdTe samples doped with various contents of Cl. The decrease in lifetime at low temperatures is due to trapping of positrons at negatively charged acceptors.

Independent of the $\mathrm{Cl}$ doping, the crystals show high resistivity. When comparing the $A$ centre concentration and the $\mathrm{Cl}$ content in CdTe:Cl (see Table) one notes that they are of the same order of magnitude, they approach each other for the $3000 \mathrm{ppm}$ sample. $\mathrm{Cl}$ on Te site is a shallow donor with a binding energy of $14 \mathrm{meV}$, the $A$ centres shallow acceptors with a binding energy of $125 \mathrm{meV}$. Since 
TABLE

Comparison of the $\mathrm{Cl}$ content added to the melt [Cl], the donors expected to be built in $\left[\mathrm{Cl}_{\mathrm{Te}}\right.$ ] with $k=3 \times 10^{-3}$ and the $A$ centre concentration [ $A$ centre] obtained by positron annihilation. For $[A$ centre] Eq. (1) was evaluated using the corresponding $\kappa_{d 2}$ and a trapping coefficient of $\mu=9 \times 10^{14} \mathrm{~s}^{-1}$.

\begin{tabular}{r|c|c|c|c}
\hline \hline Sample & {$[\mathrm{Cl}]\left[\mathrm{cm}^{-3}\right]$} & {$\left[\mathrm{Cl}_{\mathrm{Te}}\right]\left[\mathrm{cm}^{-3}\right]$} & $\kappa_{d 2}\left[\mathrm{~s}^{-1}\right]$ & {$[A$ centre $]\left[\mathrm{cm}^{-3}\right]$} \\
\hline $100 \mathrm{ppm}$ & $1.5 \times 10^{18}$ & $4.5 \times 10^{15}$ & $2.2 \times 10^{9}$ & $3.6 \times 10^{16}$ \\
$300 \mathrm{ppm}$ & $4.5 \times 10^{18}$ & $1.4 \times 10^{16}$ & $3.2 \times 10^{9}$ & $5.2 \times 10^{16}$ \\
$1000 \mathrm{ppm}$ & $1.5 \times 10^{19}$ & $4.5 \times 10^{16}$ & $5.1 \times 10^{9}$ & $8.3 \times 10^{16}$ \\
$2000 \mathrm{ppm}$ & $3.0 \times 10^{19}$ & $9.0 \times 10^{16}$ & $3.9 \times 10^{9}$ & $6.4 \times 10^{16}$ \\
$3000 \mathrm{ppm}$ & $4.5 \times 10^{19}$ & $1.4 \times 10^{17}$ & $7.9 \times 10^{9}$ & $1.3 \times 10^{17}$
\end{tabular}

undoped CdTe is usually $p$-type due to the presence of residual extrinsic impurities acting as acceptors $(\mathrm{Ag}, \mathrm{Cu}, \mathrm{Na}, \mathrm{P}, \ldots$ ) the high resistivity in $\mathrm{CdTe}: \mathrm{Cl}$ can hardly be explained by the compensation of $\mathrm{Cl}_{\mathrm{Te}}$ and the $A$ centres [7]. The resistivities found ranged from $10^{8}$ to $10^{9} \Omega \mathrm{cm}[8]$.

However, even higher values could be obtained in CdTe by doping or co-doping with the transition metal element vanadium. For vanadium doped CdTe this could be understood in the light of recent photo-EPR investigations placing the $V^{3+/ 2+}$ level at $E_{\mathrm{CB}}-0.67 \mathrm{eV}$ [9]. In $\mathrm{Cl}$ doped CdTe as well as in undoped crystals a trap at mid gap could very often be observed by electrical methods. It is most likely of intrinsic origin, but a definite assignment to a certain defect is not yet possible. One can speculate about the presence of $\mathrm{Cd}$ interstitials as well as of $\mathrm{Te}$ on $\mathrm{Cd}$ site antisite defects. Both are donor type defects and could be responsible for the compensation behaviour, but further results of experiments have to be awaited before conclusions can be drawn.

\section{References}

[1] P. Emanuelsson, P. Omling, B.K. Meyer, M. Wienecke, M. Schenk, Phys. Rev. B 47, 15578 (1993).

[2] B.K. Meyer, P. Omling, E. Weigel, G. Müller-Vogt, Phys. Rev. B 46, 15135 (1992).

[3] M. Hage-Ali, P. Siffert, Nucl. Instrum. Methods Phys. Res, A 322, 313 (1992).

[4] D.M. Hofmann, P. Omling, H.G. Grimmeiss, B.K. Meyer, K.W. Benz, D. Sinerius, Phys. Rev. B 45, 6247 (1992).

[5] C. Gely-Sykes, C. Corbel, R. Triboulet, Solid State Comm. 80, 79 (1991).

[6] R. Krause, A. Klimakow, F.M. Kiessling, A. Polity, P. Gille, M. Schenk, J. Cryst. Growth 101, 512 (1990).

[7] B.K. Meyer, D.M. Hofmann, W. Stadler, M. Salk, C. Eiche, K.W. Benz, Mat. Res. Soc. Symp. Proc. 302, 189 (1993).

[8] D. Sinerius, Ph.D. Thesis, University of Freiburg, Freiburg 1992.

[9] P. Christmann, B.K. Meyer, J. Kreissl, R. Schwarz, K.W. Benz, submitted to Appl. Phys. Lett. 